\title{
Succinate-treated macrophages attenuate dextran sodium sulfate colitis in mice
}

\author{
I Seul Park ${ }^{1,2}$, Mijeong Son ${ }^{1,2}$, Hyun Woo Ma ${ }^{1,2}$, Jihyung Kim ${ }^{1,2}$, Da Hye Kim ${ }^{1}$, Seung Won Kim ${ }^{1,2,3}$, Jae Hee Cheon ${ }^{1,2,3}$ \\ ${ }^{1}$ Department of Internal Medicine and Institute of Gastroenterology, ${ }^{2}$ Brain Korea 21 PLUS Project for Medical Science, and ${ }^{3}$ Severance \\ Biomedical Science Institute, Yonsei University College of Medicine, Seoul, Korea
}

Inflammatory bowel disease (IBD), which includes Crohn's disease and ulcerative colitis, is a chronic and inappropriate inflammatory disorder of the intestine. It is considered that interactions between genetic factors, microbiota, and host immune system are involved in the pathogenesis of IBD. Particularly, recent studies have shown that metabolites from intestinal microbes or host modulate the immune system in colitis. ${ }^{1}$ This study focused on macrophages and the role of succinate as an anti-inflammatory metabolite in IBD. Macrophages exist in almost all organs and are responsible for the first line of antimicrobial defense. ${ }^{1}$ The macrophages can be divided into pro-inflammatory M1 macrophages, called "classical macrophage," and anti-inflammatory M2 macrophages, called "alternative macrophage." It was reported that switching between M1 and M2 macrophages occurs through metabolic changes. In M2 macrophages, the succinate is oxidized to fumarate, resulting in electron transport chain flow that provides energy for adenosine triphosphate synthase. On the other hand, reverse electron transport occurs and succinate is accumulated in M1 macrophages. ${ }^{2,3}$ If the polarization of macrophages is not properly regulated, this increases the proportion of M1 mac-

Received June 23, 2020. Revised July 24, 2020. Accepted July 25, 2020. Correspondence to Jae Hee Cheon, Department of Internal Medicine, Yonsei University College of Medicine, 50-1 Yonsei-ro, Seodaemun-gu, Seoul 03722, Korea. Tel: +82-2-2228-1990, Fax: +82-2-393-6884, E-mail: GENIUSHEE@yuhs.ac

Co-Correspondence to Seung Won Kim, Severance Biomedical Science Institute, Yonsei University College of Medicine, 50-1 Yonsei-ro, Seodaemungu, Seoul 03722, Korea. Tel: +82-2-2228-0788, Fax: +82-2-2228-2812, E-mail:swk21c@hanmail.net rophages and can affect disease development such as IBD. ${ }^{4}$

Although there is a prior study using trinitrobenzene sulfonic acid-induced colitis and focusing on succinate receptor 1 (SUCNR1) and fibrosis, ${ }^{5}$ it is not yet clear whether succinate directly affects macrophage activity in colitis. Wang et al. ${ }^{6}$ showed macrophages from peritoneal cavity cells (PCCs) affect experimental colitis. Hence, to identify the direct effect of succinate on macrophages, we performed a transfer experiment to find out the effects of succinate on macrophages in a dextran sulfate sodium (DSS) (MP Biomedicals, Solon, OH, USA)-induced colitis model (Fig. 1A). All experiments using animals were reviewed and approved by the Institutional Animal Care and Use Committee of Yonsei University Severance Hospital (IACUC No: 2018-0227), and all methods were performed in accordance with relevant guidelines and regulations. Eight-weekold male C57BL/6 mice were maintained in 12 hours light/ 12 hours dark cycle at $22^{\circ} \mathrm{C}$ under specific pathogen-free conditions and used as donors of peritoneal macrophages. The following groups of mice were used as donors: injected vehicle (Veh) $(\mathrm{n}=4)$; injected succinate (Suc) $(\mathrm{n}=4)$. F4/80 $/$ peritoneal macrophages were isolated using BD FACS Aria II (BD Biosciences, San Jose, CA, USA) from the collected peritoneal cells of mice which were then intraperitoneally injected with 40 $\mathrm{mM}$ succinate or not for 3 days (Fig. $1 \mathrm{~B}$ and $\mathrm{C}$ ). Isolated F $4 / 80^{+}$ cells of $2 \times 10^{5}$ were intraperitoneally injected to recipient mice in $200 \mu \mathrm{L}$ Hanks' balanced salt solution (HBSS; Thermo Fisher Scientific, Inc., Waltham, MA, USA) (day 0) and the mice were sacrificed after $2 \%$ (wt/vol) DSS in drinking water until day 6 followed by normal water administrated for 2 days. Recipient 
A

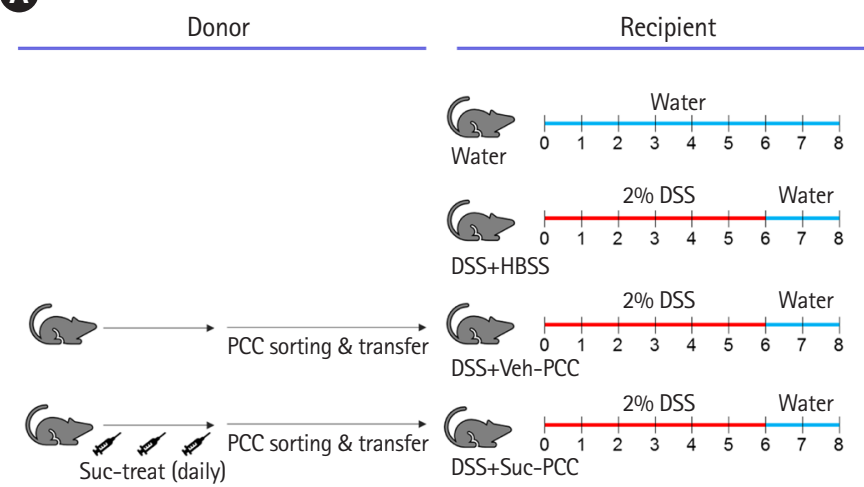

B
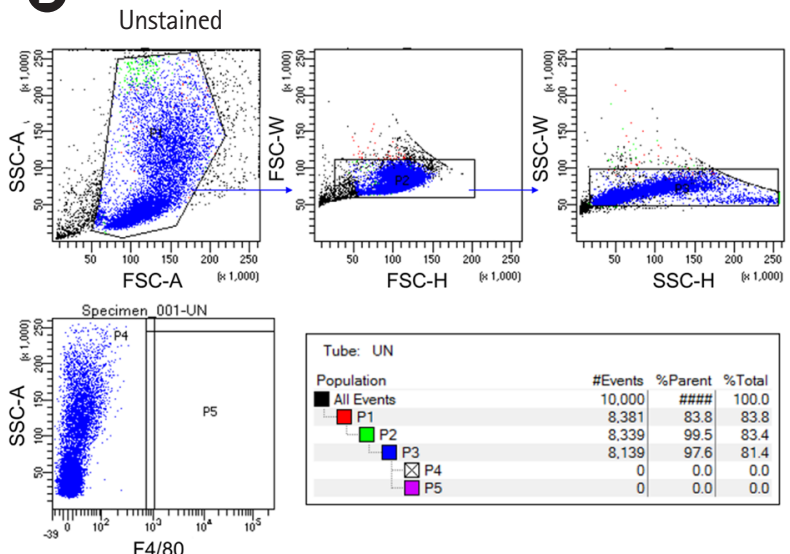

C

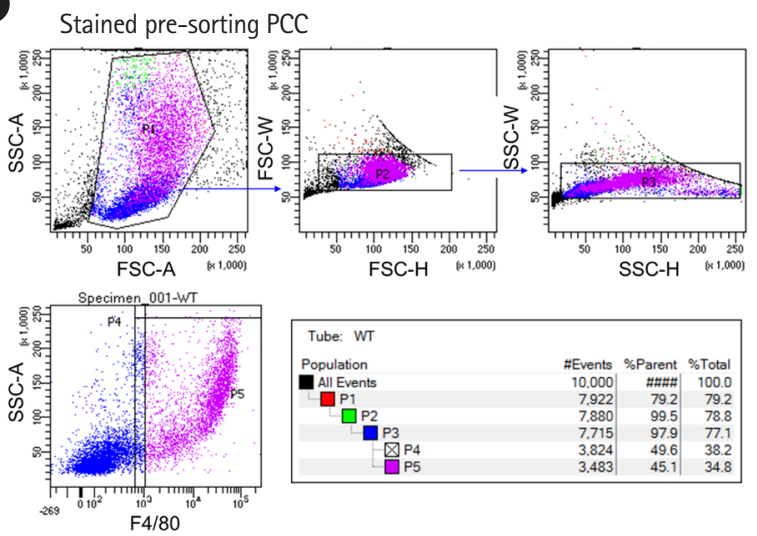

Post-sorting PCC
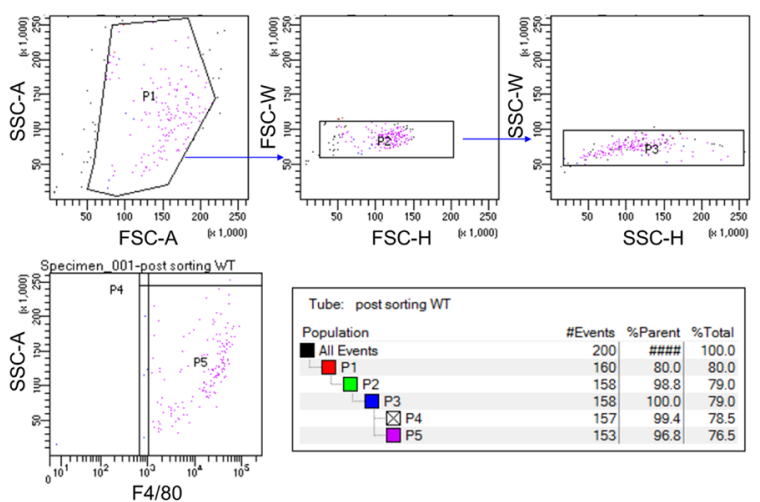

Fig. 1. Peritoneal cavity cell transfer in a dextran sulfate sodium (DSS)-colitis model. (A) Experimental design of the peritoneal cavity cells (PCCs) transfer. Donor mice were intraperitoneally injected with $40 \mathrm{mM}$ succinate or not for 3 days. Recipient mice were transferred with the PCCs on day 0 , were then administrated 2\% DSS in drinking water until day 6 followed by normal water for 2 days and were sacrificed on day 8. (B, C) Gating strategy of peritoneal macrophage sorting. F4/80+ PCCs isolation using BD FACS Aria II. (B) Unstained PCCs. (C) Stained pre-sorting PCCs and post-sorting PCCs. HBSS, Hanks' balanced salt solution; Veh, vehicle; Suc, succinate; SSC-A, side-scatter area; FSC-A, forward-scatter area; FSC-W, forward-scatter width; FSC-H, forward-scatter height; SSC-W, side-scatter width; SSC-H, sidescatter height; WT, water.

groups were as follows: water, administered with normal water ( $\mathrm{n}=3)$; DSS+HBSS, administered with HBSS ( $\mathrm{n}=6$ ); DSS+VehPCC, recipients of PCC $(\mathrm{n}=4)$; DSS+Suc-PCC, recipients of PCC treated succinate $(n=4)$. To estimate the severity of colitis, body weight, and disease activity index (DAI) were checked daily. The DAI scores, which ranged from 0 to 4 , were calculated using the following parameters: body weight loss (0, none; 1, 1\%-5\%; 2, 5\%-10\%; 3, 10\%-20\%; 4, >20\%), stool consistency (0, negative; 1 and 2, loose; 3 and 4, diarrhea), and bleeding ( 0 , absence; 1 and 2, slightly bleeding; 3 and 4, bloody). ${ }^{7}$ The calculated formula was as follows: $\mathrm{DAI}=$ (body weight loss score)+ (stool consistency score)+(bleeding score). The colon length was measured between the ileocecal junction and the proximal rectum.

DSS+Suc-PCC mice showed the attenuated body weight loss
(Fig. 2A), DAI (Fig. 2B), and a colon length shortening (Fig. 2C) compared with DSS+Veh-PCC mice. For histological analysis, segments of distal colons were embedded in paraffin and stained with periodic acid-Schiff to observe goblet cells and mucins. We found that mucin levels were significantly increased in succinate-treated PCC group compared with DSS+Veh-PCC group (Fig. 3). Taken together, these results suggest that succinatetreated PCC attenuates DSS-induced colitis.

Although some researches on succinate have mainly described succinate as a pro-inflammatory factor in many inflammatory diseases, ${ }^{8}$ there are contradictory studies that succinate acts as an anti-inflammatory factor., ${ }^{910}$ The succinate secreted by the cancer cells into their microenvironment induces polarization into tumor-associated macrophages through SUCNR1 signaling of macrophages. ${ }^{9}$ Besides, another study showed 

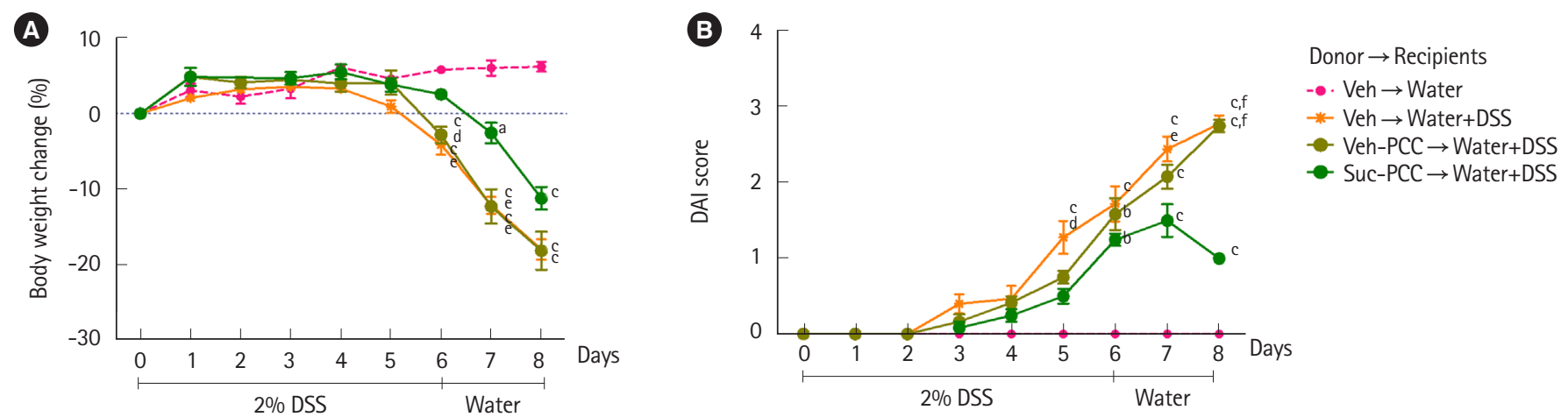

C
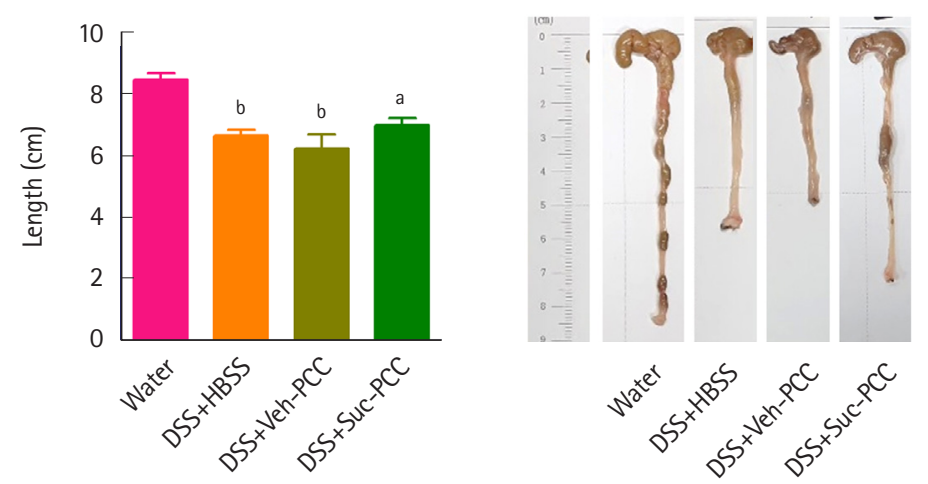

Fig. 2. Transferred succinate-treated peritoneal cavity cells (Suc-PCCS) ameliorate dextran sulfate sodium (DSS)-induced colitis. The recipients of Suc-PCCs show the attenuated body weight change (A), disease activity index (DAI) (B), and colon length (C) compared to vehicle-treated PCC (Veh-PCC). The results were examined using Prism 5.0 software (GraphPad Inc.). Statistical significance was evaluated using one-way analysis of variance and $P<0.05$ were regarded to be significant. Bars in graphs represent mean \pm standard error of the mean. ${ }^{a} P<0.05,{ }^{b} P<0.01$, and ${ }^{c} P<0.001$ compared with the water group. ${ }^{d} P<0.05$, ${ }^{e} P<0.01$, and ${ }^{f} P<0.001$ compared with the Suc-PCC group. HBSS, Hanks' balanced salt solution.

that SUCNR1 on macrophages plays a role in inducing antiinflammatory phenotype and limiting inflammation. ${ }^{10}$ These controversial results showing pro- and anti-inflammatory effects of succinate might be due to the complexity of the immune system. In this study, we addressed the protective function of succinate in macrophages.

It is well known that succinate dehydrogenase is impaired in inflammatory conditions, and then succinate is accumulated. Further studies investigating the effects of succinate on various macrophages types, using cell lines and primary cells from SucnrI ${ }^{-1}$ mice would be warranted.

\section{ADDITIONAL INFORMATION}

\section{Funding Source}

This work was supported by Mid-career Researcher Program through NRF grant funded by the Korea government (MSIP) (NRF-2017R1A2B4001848) and by the Research Program funded by the Korea Centers for Disease Control and Preven- tion (2019-ER6905-01).

\section{Conflict of Interest}

Cheon JH is an editorial board member of the journal but was not involved in the peer reviewer selection, evaluation, or decision process of this article. No other potential conflicts of interest relevant to this article were reported.

\section{Author Contribution}

Conceptualization: Kim SW, Cheon JH. Data curation: Park IS, Kim SW, Ma HW. Formal analysis: Park IS, Son M. Funding acquisition: Cheon JH. Investigation: Park IS, Son M, Kim J. Methodology: Kim SW, Kim DH, Ma HW. Project administration: Kim SW, Cheon JH. Resources: Kim SW, Cheon JH. Software: Park IS, Kim SW. Supervision: Kim SW, Cheon JH. Validation: Kim SW, Cheon JH. Visualization: Park IS. Writing - original draft: Park IS. Writing - review \& editing: Kim SW, Cheon JH. Approval of final manuscript: all authors. 


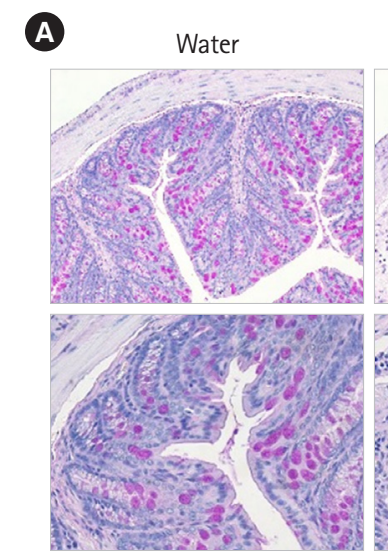

B

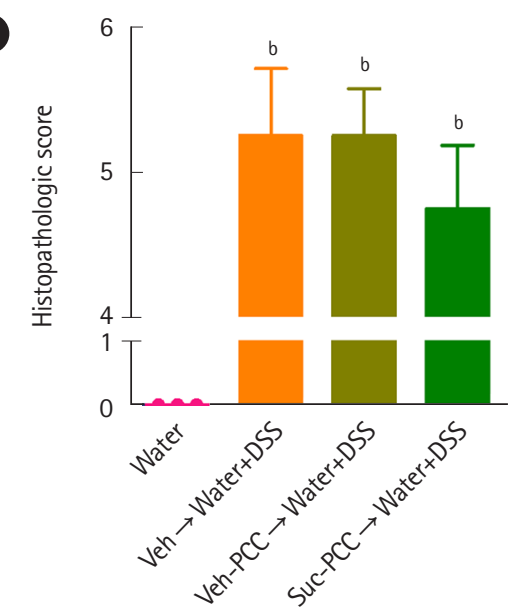

DSS+HBSS
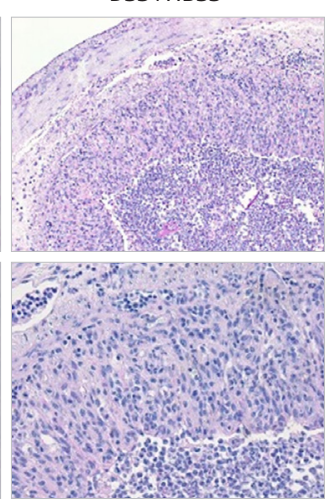
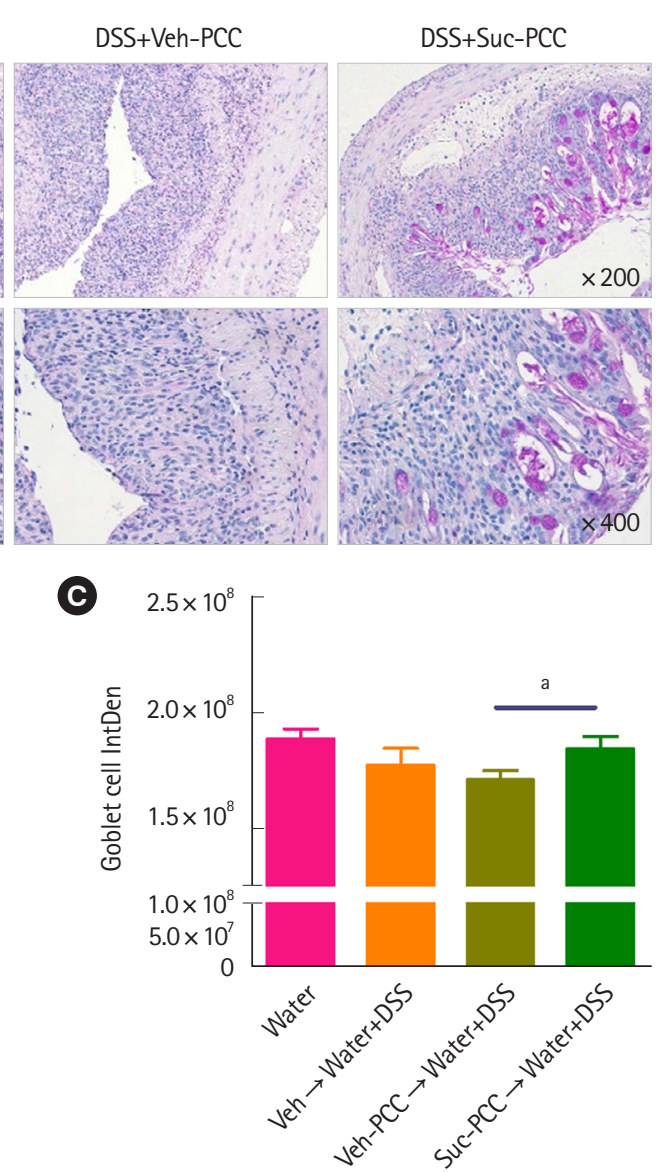

Fig. 3. Periodic acid Schiff staining and quantification of colitis. (A) Section from colon tissues stained with periodic acid Schiff. Histopathologic scores (B) and quantification with integrated density (IntDen) of goblet cells (C) of colon tissues from mice were assessed by Image J v.1.496 software (National Institutes of Health). The results were examined using Prism 5.0 software (GraphPad Inc.). Statistical significance was evaluated using one-way analysis of variance and $t$-test, respectively, $P<0.05$ were considered significant. Bars in graphs represent mean \pm standard error of the mean. ${ }^{a} P<0.05,{ }^{b} P<0.001$ compared with the water group in (B). DSS, dextran sulfate sodium; HBSS, Hanks' balanced salt solution; PCC, peritoneal cavity cell; Veh, vehicle; Suc, succinate.

\section{ORCID}

Park IS

Son M

Ma HW

Kim J

Kim DH

Kim SW

Cheon JH https://orcid.org/0000-0001-6546-5456

https://orcid.org/0000-0002-5889-8488 https://orcid.org/0000-0002-3891-4495 https://orcid.org/0000-0001-5381-3628 https://orcid.org/0000-0003-0513-6583 https://orcid.org/0000-0002-1692-1192 https://orcid.org/0000-0002-2282-8904

\section{REFERENCES}

1. Lloyd-Price J, Arze C, Ananthakrishnan AN, et al. Multi-omics of the gut microbial ecosystem in inflammatory bowel diseases. Nature 2019;569:655-662.

2. Diskin C, Pålsson-McDermott EM. Metabolic modulation in macrophage effector function. Front Immunol 2018;9:270.

3. Viola A, Munari F, Sánchez-Rodríguez R, Scolaro T, Castegna A. The metabolic signature of macrophage responses. Front Immunol 2019;10:1462.

4. Mizoguchi E, Low D, Ezaki Y, Okada T. Recent updates on the basic mechanisms and pathogenesis of inflammatory bowel diseases in experimental animal models. Intest Res 2020;18: 151-167.

5. Macias-Ceja DC, Ortiz-Masiá D, Salvador P, et al. Succinate receptor mediates intestinal inflammation and fibrosis. Mucosal Immunol 2019;12:178-187.

6. Wang W, Li X, Zheng D, et al. Dynamic changes of peritoneal macrophages and subpopulations during ulcerative colitis to metastasis of colorectal carcinoma in a mouse model. Inflamm Res 2013;62:669-680. 
7. Seo DH, Che X, Kwak MS, et al. Interleukin-33 regulates intestinal inflammation by modulating macrophages in inflammatory bowel disease. Sci Rep 2017;7:851.

8. Connors J, Dawe N, Van Limbergen J. The role of succinate in the regulation of intestinal inflammation. Nutrients 2018;11:25.

9. Wu JY, Huang TW, Hsieh YT, et al. Cancer-derived succinate promotes macrophage polarization and cancer metastasis via succinate receptor. Mol Cell 2020;77:213-227.

10. Keiran N, Ceperuelo-Mallafré V, Calvo E, et al. SUCNR1 controls an anti-inflammatory program in macrophages to regulate the metabolic response to obesity. Nat Immunol 2019;20: 581-592. 